\title{
Objective Selection Criteria between ALT and Radial Forearm Flap in Oral Soft Tissues Reconstruction
}

\author{
Elisa Benanti ${ }^{1}$ Marta Starnoni ${ }^{1}$ Antonio Spaggiari ${ }^{1}$ Massimo Pinelli ${ }^{1}$ Giorgio De Santis ${ }^{1}$ \\ ${ }^{1}$ Department of Plastic and Reconstructive Surgery, Policlinico di \\ Modena, University of Modena and Reggio Emilia, Modena, Italia

\begin{abstract}
Address for correspondence Elisa Benanti, MD, Policlinico di Modena 71 Largo del Pozzo, 41125 Modena MO, Italia (e-mail: elisa.benanti@gmail.com).
\end{abstract}

Indian J Plast Surg 2019;52:166-170

\begin{abstract}
Different locoregional and free flaps were described for oral soft tissues reconstruction after oncological resections; however, free flaps remain the first choice. Among free flaps, the radial forearm flap (RFF) and the anterolateral thigh perforator flap (ALT) are preferred the most. The lack of standardization of the flap choice leaves the selection to the surgeon's experience. The purpose of our observational study is to provide an algorithm to support the flap choice for the reconstruction of oral soft tissues. Sixty patients with squamous cell carcinoma of oral soft tissues were enrolled in our study. All the patients underwent preoperative magnetic resonance imaging (MRI) to measure the three-dimensional size of the tumor. During the follow-up, the patients were evaluated by using the University of Washington-Quality of Life Questionnaire. The

\section{Keywords}

- oral soft tissue reconstruction

- radial forearm flap

- anterolateral thigh flap

- flap selection questionnaire score was better for small tumors and worse for large tumors in both functional and relational fields. We observed that most of the overlapping results were obtained for small defects and the choice of RFF, as well as for large defects and the use of ALT. We observed that in the preoperative time, it is possible to select which flap between radial forearm and ALT is more appropriate for oral soft tissues defects reconstruction, according to the size of the tumor evaluated by MRI. We propose a decisional algorithm that suggests the type of flap to use between ALT and RFF.
\end{abstract}

\section{Introduction}

Oral soft tissue defects need an appropriate treatment in order to maintain the proper integrity of orodigestive tract and allow life functions, such as chewing, swallowing, speech, and facial expression.

Free flaps are the gold standard for head and neck reconstruction, ${ }^{1}$ especially after tumor resection. ${ }^{2}$ Despite the higher costs compared to the pedicled flaps ${ }^{3}$ (including longer surgical time, patients monitoring, intensive care, and hospitalization time), free flaps remain the first choice for better tissue match related to form and function, ${ }^{4}$ the better quality of life ${ }^{5}$ offered to the patients, and the longer term of survival, probably due to better margins resection. ${ }^{6}$

Most of the patients need to undergo radiation therapy and the use of vascularized tissues allows to achieve better results $^{7}$; however, pedicled flaps remain a valid option when recipient vessels do not permit good anastomosis. ${ }^{8}$ The radial forearm flap (RFF), rectus abdominis, and free anterolateral thigh perforator flap (ALT) are the most used free flaps for soft tissue reconstruction. When bone reconstruction is required, ${ }^{9}$ the RFF along with radius and the fibula flaps are the most suitable options. The RFF is the most suitable for oropharynx reconstruction because of its long pedicle and the pliable skin paddle which can be modeled to fix the tongue base, tonsillar fossa, soft palate, and posterior floor of the mouth. ${ }^{4}$

In head and neck reconstruction, the ALT flap finds its indication in the possibly large size of the skin paddle. ${ }^{4}$ In most cases, the flap selection is related to the dimension of the defect and the tissue components ${ }^{4}$; however, the lack of a standardized procedure leaves the choice to the experience and preference of the surgeon. ${ }^{10}$

In this article, we would like to present our algorithm for choosing the free flap in the reconstruction of the oral soft published online September 30, 2019
DOI https://doi.org/

10.1055/s-0039-1693504 ISSN 0970-0358.
(C)2019 Association of Plastic Surgeons of India
License terms

(이 (1) $\Theta \circledast$ 
tissue based on the extent of the defect and on the area that needs to be reconstructed. We analyzed, retrospectively, 60 free flaps that have been used in oral soft tissues reconstruction. We observed that in most cases, the choice was between radial forearm and the ALT flap (extensive reconstructions with muscular flaps were excluded) depending on surgeons' experience.

\section{Subjects and Methods}

Sixty patients with squamous cell carcinoma of oral soft tissues were enrolled in our study. The age of the patients was between 25 and 50 years old, the body mass index (BMI) between 18 and $30 \mathrm{~kg} / \mathrm{m}^{2}$. In this observational study, we included patients without metastasis, who underwent radiotherapy after surgery. Exclusion criteria were cardiopulmonary disease or other major general morbidity and major obesity. The week before surgery, all the patients underwent preoperative magnetic resonance imaging (MRI) which allowed us to measure the three dimensions of the tumor. The patients were divided into three groups based on tumor's location: 20 patients with tumors mainly involving the floor of the mouth, 20 primarily involving the tongue, and 20 involving the retromolar trigone extending to the tonsillar area.

We have grouped the patients according to the most involved area because it is unusual that a single area is affected. For each group, 10 reconstructions with ALT flaps and 10 reconstructions with RFFs were considered for tumors of different sizes. Total glossectomies were excluded.

The surgeries were conducted from 2014 to 2017. The average follow-up was 2 years (considering a minimum of 1 year and a maximum of 3 years after surgery). During the follow-up the patients were evaluated by using the University of Washington Quality of Life Questionnaire (UW-QOL)) which allowed us to assess physical and functional outcomes and the quality of life. The results of the questionnaire were analyzed by assessing for each group of patients (for each area reconstructed) the extent of the tumor measured by the preoperative MRI and the flap used (ALT or RFF) according to the preference of the surgeons.

Concerning the preoperative dimension of the tumor we defined "small" a tumor with a calculated volume $<50 \mathrm{cc}$, "large" with a volume $>70 \mathrm{cc}$, and "border" between 50 and $70 \mathrm{cc}$.

\section{Results}

Different results were shown according to the reconstructed area:

Group 1: patients with tumors mainly involving the floor of the mouth. The average score was 911.25 with a minimum of 445 and a maximum of 1,245 . The majority of patients indicated the variable "chewing" as the major dysfunction among physical defects and the variable "overall quality of life during the past 7 days" among the global questions.

Group 2: patients with tumors mainly involving the tongue. The average score was 760.5 with a minimum of
310 and a maximum of 1,155 . The majority of patients indicated the variable "speech" as the major dysfunction among the physical defects and the variable "health-related quality of life (QOL) compared to month before had cancer" among the global questions.

Group 3: patients with tumors mainly involving the retromolar trigone extending to the tonsillar area. The average score was 878.5 with a minimum of 430 and a maximum of 1,120 . The majority of patients indicated the variable "swallowing" as the major dysfunction among the physical defects and the variable "health-related QOL compared to month before had cancer" among the global questions. Among the different groups, reconstruction of the floor obtained, on average, the best results and, the tongue, the worst.

Analyzing the size of the tumor, we observed that the questionnaire score was better for small tumors and worse for large tumors in both functional and relational fields in all the groups. Analyzing, then, the type of flap used for each category, we observed that most of the overlapping results (good and not so good) were obtained for small defects $(<50)$ and the choice of RFF, as well as for large defects $(>70)$, and the use of ALT. Observing the average distribution score of the questionnaires for both small and large tumors, we noticed that there were some cases that diverged from the average showing worse results.

The Figures of each group show five cases in group 1 , seven in group 2, and four in group 3 in which the questionnaire scores do not overlap with the average of other cases with tumors of similar dimension, but are much lower than average. ( - Fig. 1-3)

In these 11 cases, the correspondence between tumor size and used flap was not observed.

In group 1: three cases had a tumor size $>70 \mathrm{cc}$ and RFF was used; two cases had tumor size $<50 \mathrm{cc}$ and ALT flap was used.

In group 2: four cases had tumor size < 50 cc with ALT flap and three cases a size $>70 \mathrm{cc}$ with RFF.

In group 3: one case $>70 \mathrm{cc}$ with RFF used and three cases $<50$ cc with ALT flap.

Eight cases had tumor size between $50 \mathrm{cc}$ and $70 \mathrm{cc}$ and they all achieved good results regardless of the flap: five cases with ALT flap reconstruction and three with RFF with a score that overlaps the average of good scores for each category. In these five cases with ALT reconstruction, the patients had a $\mathrm{BMI}<25 \mathrm{~kg} / \mathrm{m}^{2}$.

\section{Discussion}

Our retrospective study showed that the results of UW-QOL were worse for the group 2 (patients whose tumors mainly affected the tongue) and better for the group 1 (patients whose tumors mainly affected the floor).

We observed that in the preoperative time, it is possible to select which flap, radial forearm or ALT, is more appropriate for oral soft tissues defects reconstruction according to the dimension of the tumor evaluated by the magnetic resonance. The UW-QOL was used to evaluate the outcomes.

Hassan and Weymuller first described the UW-QOL. ${ }^{11}$ This questionnaire allows us to assess the quality of life of patients 


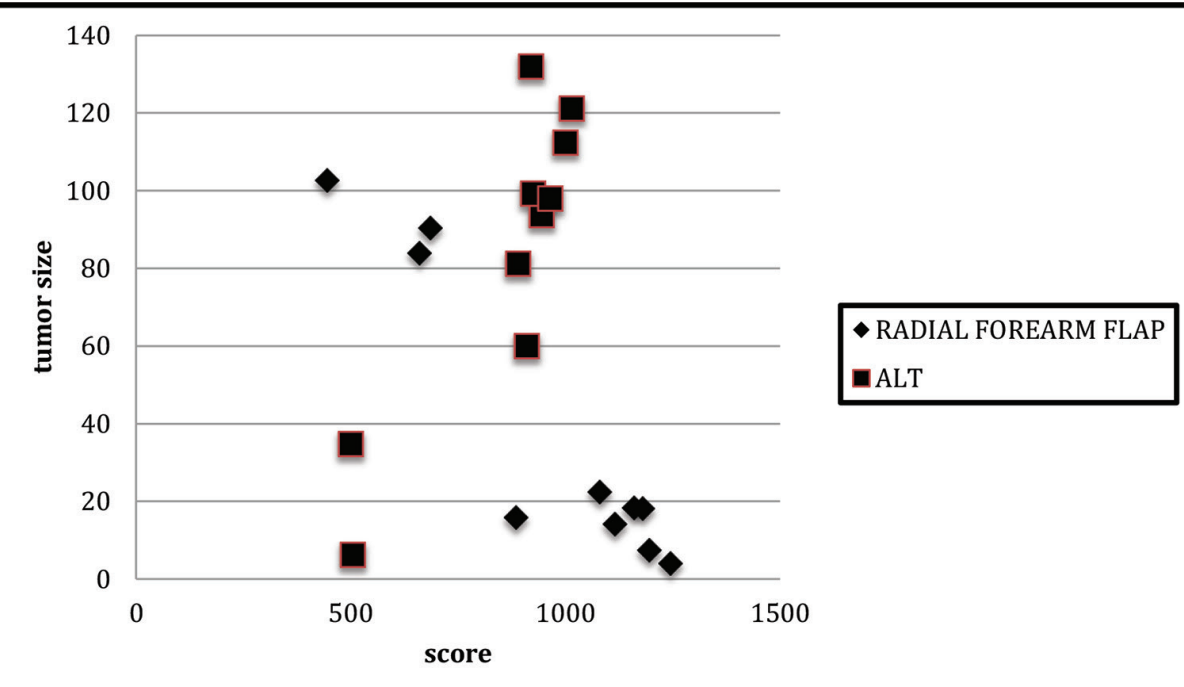

Fig. 1 Group 1 distribution: patients with tumors mainly involving the floor of the mouth.

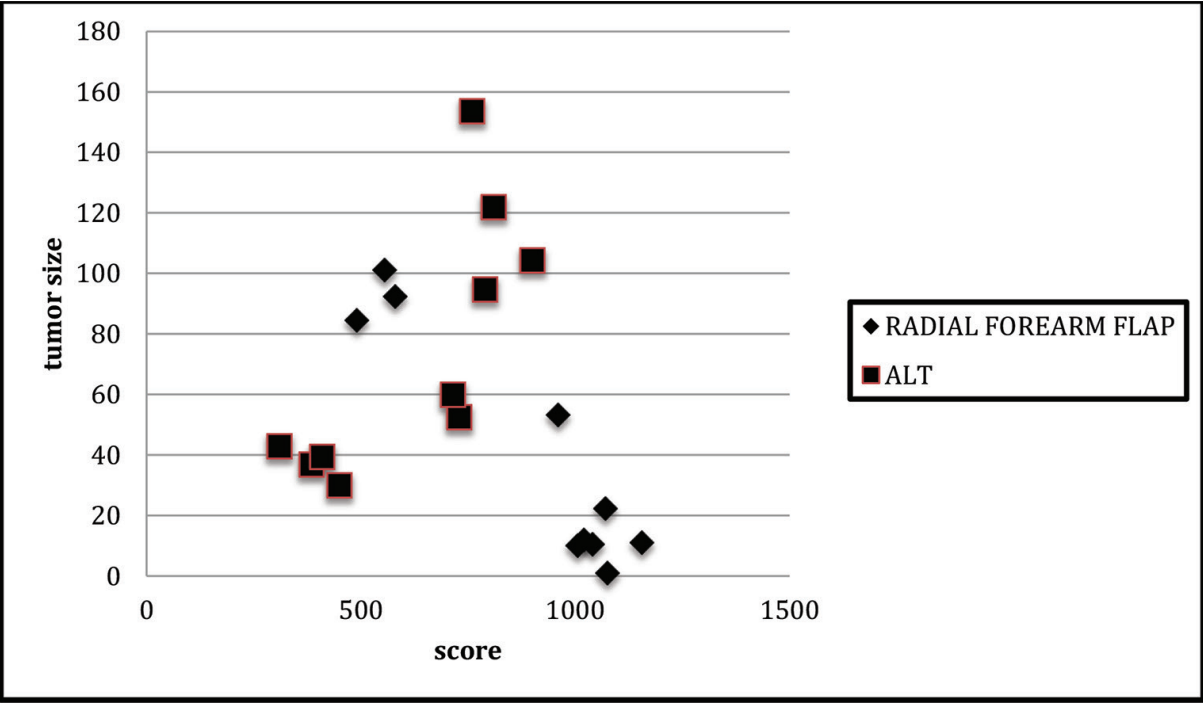

Fig. 2 Group 2 distribution: patients with tumors mainly involving the tongue.

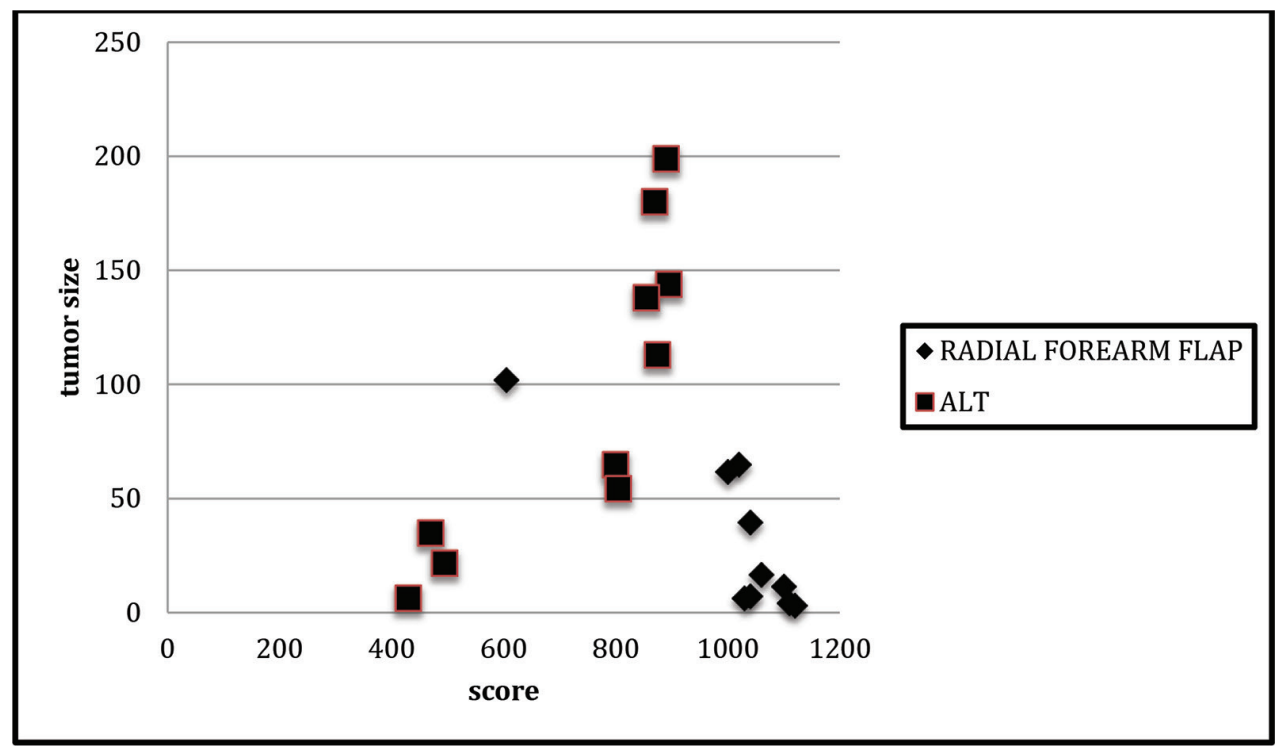

Fig. 3 Group 3 distribution: patients with tumors mainly involving the retro-molar trigone extending to the tonsillar area. 
undergoing cancer surgery in the head and neck area. The questionnaire consists of 12 questions with three to six possible answers and a score is assigned to each answer. The score is scaled in such way, so that a score of 0 represents the worst possible response and a score of 100 represents the best possible response. The questions concern pain, appearance, activity, recreation, swallowing, chewing, speech, shoulder, taste, saliva, mood, and, therefore, include both physical and mental health assessment. Three other global questions complete the questionnaire, one on how patients feel compared to life before the onset of the cancer, one on their health-related QOL, and last one on their overall QOL. This questionnaire allowed us to obtain an overall assessment on the patient. ${ }^{11}$

In our study, we observed that the size of the tumor, evaluated with preoperative MRI, is an indicator for the type of flap to be chosen between ALT and radial forearm flap. Patients with $<50 \mathrm{cc}$ tumour volume by MRI reconstructed with RFF and patients with $>70 \mathrm{cc}$ vol. reconstructed with ALT flap obtained better results in the questionnaire.

In the border area between 50 and $70 \mathrm{cc}$, according to our experience, we used radial flaps if the patient's BMI is $>25 \mathrm{~kg} / \mathrm{m}^{2}$, while we used ALT if $<25$; therefore, preferring the ALT flap, when possible, because it has less morbidity at the donor site.

Among the head-neck tumors, squamous cell carcinoma (SCC) is the most frequent. The management of these tumors requires a multidisciplinary approach mainly with oncologists, surgeons, and radiotherapists. In most cases, squamocellular carcinomas arise from the posterior third of the tongue, from the tonsillar trigone region, the soft palate, and the posterior pharynx.

For advanced locoregional tumors, the standardized approach includes surgical resection and postoperative radiotherapy, with or without chemotherapy. Early-stage tumors may have a single approach with surgery or radiation therapy. In any case, more treatment options are developing. ${ }^{12}$ The purpose of postoncological reconstruction is mainly functional: the challenge is to restore the shape, size, type of tissue of the defect, and to achieve functions that allow a good quality of life, such as eating, speaking, swallowing, etc., and avoid complications, such as fistulas.

Among the reconstructive options, there are both locoregional and free flaps. The usual tendency is to use free tissue transfer instead of locoregional flap which lead to more complications, such as necrosis, dehiscence, and fistula formation, ${ }^{13}$ and they do not restore a good tissue matching and often result in function. Regional flaps, such as the pectoralis major and the deltopectoral, may be effective in providing good tissues but they are not generally considered as the first choice. ${ }^{14}$

The use of free flaps for upper aerodigestive tract reconstruction has yielded excellent results in terms of speaking, swallowing, and other functions of daily life. ${ }^{15}$ Free flaps for intraoral reconstruction have been described since 1976. ${ }^{16}$ The ALT and RFFs are the most used flaps for soft tissues reconstruction in head and neck. ${ }^{17}$
The RFF, described in $1981,{ }^{18}$ allows a good reconstruction of soft tissue defects thanks to its pliability ${ }^{19}$ and it can be harvested as a large, thin, and pliable flap with excellent reliability and simplicity of harvest. ${ }^{20}$ The main disadvantage of this flap is the poor donor site appearance when skin grafting is required.

The ALT flap, described in $1984,{ }^{21}$ offers many reconstructive possibilities in the head and neck area with the advantage of leaving few morbidity at the donator site and it provides very large tissue volumes and is versatile. ${ }^{22}$ However, ALT flap is difficult to use in patients with a large subcutaneous thickness of the thigh (in obese patients) and the delicate perforating vessels, ${ }^{4}$ along with its difficult defatting, limit its use according to surgeon's experience. Furthermore, if a large flap is needed, a skin graft is required for donor site closure. Both these flaps are well suitable for tissues, such as the oral cavity, allowing a good restoration of the function after oncological resection. ${ }^{14}$

The RFF has traditionally been the flap of choice for reconstructing partial or hemiglossectomy defects, ${ }^{23}$ but, as already mentioned, the ALT flap in some cases has replaced the radial forearm for intraoral reconstruction, including the reconstruction of hemiglossectomy defect. ${ }^{6}$ From a functional point of view, the most difficult area to repair is the posterior tongue that allows normal movement of the epiglottis and maintains swallowing and speaking functions. ${ }^{14} \mathrm{~A}$ decisional algorithm on the use of ALT or RFF has never been standardized in literature.

Some authors use the RFF as the first choice for defects of the tongue $<80 \%$ and the ALT flap for a nearly total glossectomy. Concerning pharyngeal defects, the authors consider the possibility of indifferent use of ALT or radial flap if the defect is $>3 \mathrm{~cm} .{ }^{24}$ Neligan et al base their choice on the thickness of the thigh: for patients with an excess of subcutaneous tissue on the thigh, the choice falls on the RFF. ${ }^{25}$ Huang and colleagues prefer ALT flap for tongue reconstruction due to the greater donor site morbidity of the RFF. ${ }^{26}$ According to Gurtner, unlike the RFF which is perfect for partial tongue defects up to $70 \%$, both the rectus abdominis and ALT free flaps can provide sufficient volume for nearly total or total glossectomy defects. ${ }^{26}$

However, the lack of standardization for the use of a specific flap leaves the choice to the surgeon's experience and preference. ${ }^{10}$ In our retrospective study, we analyzed the oral soft tissues reconstructions after tumor resections and we propose a decisional algorithm that suggests the type of flap to use between ALT and RFF.

We consider choosing RFF for defects $<50 \mathrm{cc}$ and ALT $>70$ cc evaluating at the preoperative MRI. For defects between $50 \mathrm{cc}$ to $70 \mathrm{cc}$, we refer to patients' BMI. (-Fig. 4).

This algorithm can be considered as a decisional beginning, especially for young surgeons who are approaching this surgery. However, we do not consider it as a dogma and the surgeon's experience must be always taken into consideration. 


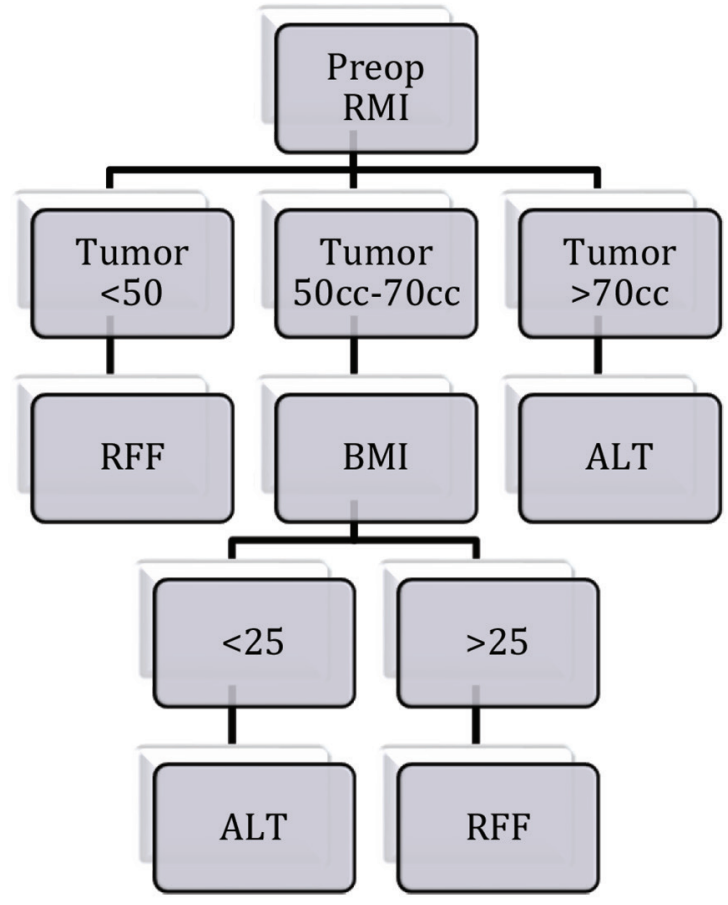

Fig. 4 Algorithm showing flap selection.

\section{Conflict of Interest}

None.

\section{References}

1 Wong CH, Wei FC. Microsurgical free flap in head and neck reconstruction. Head Neck 2010;32(9):1236-1245

2 Nakatsuka T, Harii K, Asato H, et al. Analytic review of 2372 free flap transfers for head and neck reconstruction following cancer resection. J Reconstr Microsurg 2003;19(6):363-368, discussion 369

3 McCrory AL, Magnuson JS. Free tissue transfer versus pedicled flap in head and neck reconstruction. Laryngoscope 2002;112(12):2161-2165

4 Smith RB, Sniezek JC, Weed DT, Wax MK; Microvascular Surgery Subcommittee of American Academy of Otolaryngology-Head and Neck Surgery. Utilization of free tissue transfer in head and neck surgery. Otolaryngol Head Neck Surg 2007;137(2):182-191

5 Li W, Yang Y, Xu Z, et al. Assessment of quality of life of patients with oral cavity cancer who have had defects reconstructed with free anterolateral thigh perforator flaps. Br J Oral Maxillofac Surg 2013;51(6):497-501

6 de Vicente JC, de Villalaín L, Torre A, Peña I. Microvascular free tissue transfer for tongue reconstruction after hemiglossectomy: a functional assessment of radial forearm versus anterolateral thigh flap. J Oral Maxillofac Surg 2008;66(11):2270-2275

7 Ariyan S, Ross DA, Sasaki CT. Reconstruction of the head and neck. Surg Oncol Clin N Am 1997;6(1):1-43
8 Blackwell KE, Buchbinder D, Biller HF, Urken ML. Reconstruction of massive defects in the head and neck: the role of simultaneous distant and regional flaps. Head Neck 1997;19(7):620-628

9 Disa JJ, Pusic AL, Hidalgo DH, Cordeiro PG. Simplifying microvascular head and neck reconstruction: a rational approach to donor site selection. Ann Plast Surg 2001;47(4):385-389

10 Thiele OC, Seeberger R, Engel M, Freier K, Hoffmann J. Development of the clinical use of distant flaps for head and neck reconstruction. J Craniomaxillofac Surg 2014;42(1):79-83

11 Hassan SJ, Weymuller EA, Jr. Assessment of quality of life in head and neck cancer patients. Head Neck 1993;15(6):485-496

12 Hay A, Nixon IJ. Recent advances in the understanding and management of oropharyngeal cancer. F1000 Res 2018;7:7

13 Mehta S, Sarkar S, Kavarana N, Bhathena H, Mehta A. Complications of the pectoralis major myocutaneous flap in the oral cavity: a prospective evaluation of 220 cases. Plast Reconstr Surg 1996;98(1):31-37

14 Ragbir M, Brown JS, Mehanna H. Reconstructive considerations in head and neck surgical oncology: United Kingdom National Multidisciplinary Guidelines. J Laryngol Otol 2016; 130(S2):S191-S197

15 Vos JD, Burkey BB. Functional outcomes after free flap reconstruction of the upper aerodigestive tract. Curr Opin Otolaryngol Head Neck Surg 2004;12(4):305-310

16 Harashina T, Fujino T, Aoyagi F. Reconstruction of the oral cavity with a free flap. Plast Reconstr Surg 1976;58(4):412-414

17 Kim EK, Evangelista M, Evans GR. Use of free tissue transfers in head and neck reconstruction. J Craniofac Surg 2008;19(6):1577-1582

18 Yang GF, Chen PJ, Gao YZ, et al. Forearm free skin flap transplantation: a report of 56 cases. 1981. Br J Plast Surg 1997;50(3):162-165

19 Chim H, Salgado CJ, Seselgyte R, Wei FC, Mardini S. Principles of head and neck reconstruction: an algorithm to guide flap selection. Semin Plast Surg 2010;24(2):148-154

20 Soutar DS, McGregor IA. The radial forearm flap in intraoral reconstruction: the experience of 60 consecutive cases. Plast Reconstr Surg 1986;78(1):1-8

21 Song YG, Chen GZ, Song YL. The free thigh flap: a new free flap concept based on the septocutaneous artery. Br J Plast Surg 1984;37(2):149-159

22 Chana JS, Wei FC. A review of the advantages of the anterolateral thigh flap in head and neck reconstruction. Br J Plast Surg 2004;57(7):603-609

23 Stark B, Nathanson A, Hedén P, Jernbeck J. Results after resection of intraoral cancer and reconstruction with the free radial forearm flap. ORL J Otorhinolaryngol Relat Spec 1998;60(4):212-217

24 Yadav P. Recent advances in head and neck cancer reconstruction. Indian J Plast Surg 2014;47(2):185-190

25 Neligan PC. Head and neck reconstruction. Plast Reconstr Surg 2013;131(2):260e-269e

26 Huang $\mathrm{CH}$, Chen HC, Huang YL, Mardini S, Feng GM. Comparison of the radial forearm flap and the thinned anterolateral thigh cutaneous flap for reconstruction of tongue defects: an evaluation of donor-site morbidity. Plast Reconstr Surg 2004;114(7):1704-1710

27 Gurtner GC, Evans GR. Advances in head and neck reconstruction. Plast Reconstr Surg 2000;106(3):672-682, quiz 683 